\title{
Hypothalamic hamartomas
}

\author{
Howard L. Weiner, MD \\ Department of Neurosurgery, NYU Langone Medical Center, New York, New York
}

$\mathrm{H}$ YPOTHALAMIC hamartomas (HHs) have long held the fascination of neurosurgeons and epilepsy neurologists since even well before the landmark discovery, in the mid-1990s, that these focal lesions deep in the center of the brain are the source of gelastic, or "laughing," seizures. Prior to this recognition, some patients with medically intractable gelastic seizures underwent standard resective epilepsy surgery, with removal of an apparent, but incorrect, epileptic focus elsewhere in the cortex, without any relief of their seizures. Much has been learned in the last 15 years, and the HH story really reflects the thought evolution that has occurred in the epilepsy surgery field in general. Although the electroencephalogram (EEG) may show bilateral or generalized seizure activity in $\mathrm{HH}$ patients, it was only when an electrode was inserted directly into the lesion that it became clear that a focal epileptogenic, surgically targetable, lesion could be masquerading as a generalized epilepsy. This lesson has also been learned in children with focal cortical dysplasias presenting with infantile spasms and other young patients with focal hemispheric malformations exhibiting generalized EEG patterns. These observations have been major breakthroughs for patients.

The fact that we have witnessed such a large array of potentially effective neurosurgical approaches to HHs in the last few years supports the impression one has of the true challenge inherent in their treatment, as well as supporting the need for more scholarly analysis of the pros and cons of the various options: microsurgery, endoscopy, stereotactic radiosurgery, and, most recently, stereotactic laser ablation (SLA). Each technique has its proponents, based on reported advantages, and many $\mathrm{HH}$ patients have been treated with a multimodality staged strategy in order to minimize risk. SLA has generated significant interest and enthusiasm in the United States in the last few years, reflected by the sharp rise in the acquisition of this technology by many centers across the country, as a treatment of epileptic lesions in general, and $\mathrm{HH}$ in particular.
In their article on MRI-guided stereotactic radiofrequency thermocoagulation (SRT), ${ }^{1}$ Kameyama et al., from Niigata, Japan, describe a minimally invasive approach, preliminarily reported in 2007, for treating any type of $\mathrm{HH}$, with a thermal "disconnection" of the lesion using a frame-based stereotactic electrode technique. Five-millimeter spherical lesions are created when this probe is heated to $74^{\circ} \mathrm{C}$ over 60 seconds. ${ }^{1}$

This is a huge series of HHs from a single center. The authors' stated goal in the retrospective analysis of 100 cases involving patients with gelastic seizures treated over a 16 -year period, since 1997, was to determine the invasiveness and feasibility of this technique. They treated both adults $(n=30)$ and children $(n=70)$, as well as lesions of a wide range of sizes, including 15 "giant" $\mathrm{HHs}$, larger than $3 \mathrm{~cm}$ in maximum diameter. Twenty-nine patients had undergone prior treatment of some type: microsurgery, endoscopy, and/or stereotactic radiosurgery, which did not preclude SRT in this center. Thirty-two patients required repeat SRT for residual/recurrent seizures and MRI evidence of incomplete disconnection. At a median follow-up of 3 years, amazingly, $71 \%$ of patients were free of all types of seizures, $86 \%$ of those with gelastic seizures were seizure free, and 18 patients were off all antiepileptic medication. The behavioral disorders improved in all the patients who were seizure free, which was essentially the vast majority of patients. These results seem better than those reported for any other type of treatment for epilepsy, in general.

This is a very impressive single-institution series in terms of numbers of patients treated, in addition to the outstanding results, and it shows that this approach holds promise for $\mathrm{HH}$ patients. The authors demonstrate the benefit of a thoughtfully dedicated uniform approach to a neurosurgical problem. In agreement with the current thinking about the pathophysiology of $\mathrm{HH}$, and in line with the general current therapeutic philosophy of epilepsy surgery, their technique is based on disconnection 
of the lesion from the surrounding brain, rather than ablation of the entire lesion volume. The paper alludes to what is likely a steep learning curve, as well as the need for a dedicated long-term management strategy in these cases. Approximately one-third of the patients in their series required repeat procedures, some more than twice. Multiple trajectories through the brain were often necessary. Many patients suffered transient hypothalamic and supplementary motor untoward symptoms, with $2 \%$ experiencing permanent pituitary dysfunction, $7 \%$ weight gain, and $9 \%$ delayed precocious puberty. Short-term memory loss occurred in $8.6 \%$ of patients. The authors argue that this morbidity profile is similar to that reported in the published results for other HH treatment approaches. Given the need for a stereotactic frame, SRT is not used by the authors for children under the age of 2 years. Neither the time required for the procedure nor the length of hospital stay are discussed in the manuscript. It will be critical, moving forward, to compare, over the long term, the SRT approach reported here with the increasingly popular SLA technique, described in 14 patients with HH (including one who was 22 months old), 12 (86\%) of whom were seizure free at follow-up of 9 months, and all of whom were discharged home within 1 day, with minimal reported morbidity. ${ }^{2}$

http://thejns.org/doi/abs/10.3171/2015.5.JNS15785

\section{References}

1. Kameyama S, Shirozu H, Masuda H, Ito Y, Sonoda M, Akazawa K: MRI-guided stereotactic radiofrequency thermocoagulation for 100 hypothalamic hamartomas. J Neurosurg [epub ahead of print November 20, 2015. DOI: 10.3171/2015.4.JNS1582]

2. Wilfong AA, Curry DJ: Hypothalamic hamartomas: optimal approach to clinical evaluation and diagnosis. Epilepsia 54 (Suppl 9):109-114, 2013.

\section{Disclosure}

The author reports no conflict of interest.

\section{Response}

\section{Shigeki Kameyama, MD, PhD, ${ }^{1}$ Hiroshi Shirozu MD, PhD, ${ }^{1}$ Hiroshi Masuda MD, ${ }^{1}$ Yosuke Ito MD, ${ }^{1}$ Masaki Sonoda MD, ${ }^{1}$ and Kohei Akazawa, PhD²}

${ }^{1}$ Hypothalamic Hamartoma Center, Department of Functional Neurosurgery, Nishi-Niigata Chuo National Hospital; and 'Department of Medical Informatics, Niigata University Medical and Dental Hospital, Niigata, Japan

We sincerely appreciate Dr. Weiner's thoughtful comments on our paper. As he described, minimally invasive surgical procedures for $\mathrm{HH}$ represent a potential alternative to resection, ${ }^{2}$ offering the ability to achieve better seizure outcomes. In our paper, we reported excellent seizure outcomes after the uniform application of SRT for a large series of HHs from a single center. SRT has a great advantage in the unrestricted selection of candidates. We discussed that the rationale and strategy of our SRT approach as applied to $\mathrm{HH}$. We also proposed that intrinsic area of ictogenesis is located in the interface between the $\mathrm{HH}$ and the hypothalamus and that seizure propagation has a direct route to the hypothalamus. ${ }^{1}$ Therefore, disconnection of the attachment from the hypothalamus using SRT is a feasible means of controlling seizures. Given our results, SRT should be regarded as the first-line treatment option for $\mathrm{HH}$, regardless of hamartoma size.

In our series of $\mathrm{HH}$ cases, all patients routinely underwent presurgical evaluation including assessment of seizure semiology and comorbidities, video-EEG, MRI, endocrinological examination, interictal and ictal SPECT, and neuropsychological assessments during 2 weeks of hospitalization. The mean time required for SRT procedures was 3 hours, including semi-microrecording and needle biopsy, if possible, after completion of MRI-guided planning. Transient postoperative hypothalamic symptoms were carefully followed up during the first 2 weeks after surgery because our SRT method required multiple trajectories to the lesion, especially for the medium-sized and giant HHs. Therefore, our patients' length of hospital stay was routinely 4 weeks, which was a necessary and acceptable duration for patient evaluation and care, even for foreign patients. Although the frame-based stereotactic surgery required completion of the cranial suture, our youngest candidate was a 22-month-old child with precocious puberty.

In our discussion, we emphasized that functional deficiency of the supplementary motor area due to SRT of the $\mathrm{HH}$ was caused by network inactivation between the mediodorsal nucleus of the thalamus and the supplementary motor area immediately after SRT surgery. Our procedure did not directly injure those areas, and trajectories mostly passed though the unilateral genu of the internal capsule to the lesion. Voluntary facial movement was intact, while contralateral emotional facial paresis was observed after $55 \%$ of the SRT procedures. This peculiar symptom should be examined in future studies.

The recent development of SLA is very interesting, ${ }^{3}$ although the selection of the candidates, limitations of the procedure, and sizes of the lesions were not described in that study. Additionally, the exact volume of ablation and exact location of the target were unclear. It will be important to see data from a large series of patients treated with this procedure before it becomes established. Moreover, there may be a medico-economical issue with respect to SLA, as it is very expensive for patients with HH. Our SRT system is popular, less expensive, and versatile, having been initially developed for the treatment of Parkinson's disease and essential tremor.

\section{References}

1. Kameyama S, Masuda H, Murakami H: Ictogenesis and symptomatogenesis of gelastic seizures in hypothalamic hamartomas: An ictal SPECT study. Epilepsia 51:22702279, 2010

2. Quigg M, Harden C: Minimally invasive techniques for epilepsy surgery: stereotactic radiosurgery and other technologies. J Neurosurg 121 Suppl:232-240, 2014

3. Wilfong AA, Curry DJ: Hypothalamic hamartomas: optimal approach to clinical evaluation and diagnosis. Epilepsia 54 (Suppl 9):109-114, 2013 